\title{
Bevölkerungsbezogene psychische Gesundheit als Schlüsselfaktor im Umgang mit COVID-19
}

\author{
Public Mental Health as One of the Key Factors in Dealing with \\ COVID-19
}

\author{
Autoren \\ Omar Hahad1, 2, Donya A Gilan³, 4, Andreas Daiber¹, 2, Thomas Münzel1, 2
}

Institute

1 Zentrum für Kardiologie - Kardiologie I, Universitätsmedizin der Johannes Gutenberg-Universität Mainz, Mainz

2 Deutsches Zentrum für Herz-Kreislauf-Forschung (DZHK), Standort Rhein-Main, Mainz

3 Leibniz-Institut für Resilienzforschung (LIR), Mainz

4 Klinik für Psychiatrie und Psychotherapie, Universitätsmedizin der Johannes Gutenberg-Universität Mainz, Mainz

\section{Schlüsselwörter}

COVID-19, Öffentliche Gesundheit, Psychische Belastungen, Resilienz

Key words

COVID-19, Public health, Mental stress, Resilience

Bibliografie

DOI https://doi.org/10.1055/a-1160-5770

Online-Publikation: 30.4 .2020

Gesundheitswesen 2020; 82: 389-391

(c) Georg Thieme Verlag KG Stuttgart · New York

ISSN 0941-3790

\section{Korrespondenzadresse}

Dr. Omar Hahad

Center for Cardiology - Cardiology I

University Medical Center Mainz

Langenbeckstraße 1

55131 Mainz

omar.hahad@unimedizin-mainz.de

\section{ZUSAMMENFASSUNG}

Ziel Das Ziel des Beitrages besteht darin, auf die bedeutsame Rolle der Prävention und Reduktion der psychischen Belastungen in der Allgemeinbevölkerung und in sensiblen Gruppen im Rahmen der Coronavirus-Krankheit-2019 (COVID-19) -Pandemie hinzuweisen.

Methodik Der vorliegende Beitrag umfasst die Analyse und Bewertung von Studien und Empfehlungen von Organisationen wie der Weltgesundheitsorganisation (WHO), die die bevölkerungsbezogenen psychischen Auswirkungen von Epi-/Pande- mien und deren Einfluss auf den weiteren Verlauf untersucht haben.

Ergebnisse Angstbedingte Verhaltensweisen können sich negativ auf den Verlauf von Epidemien auswirken. Im Rahmen vergangener Ausbrüche von Infektionskrankheiten (Ebola- und Zika-Virus) konnte ermittelt werden, dass maladaptive Verhaltensweisen, bedingt durch erhöhte psychische Belastungen und Ängste, die Implementierung von Behandlungsstrategien und -maßnahmen beeinträchtigen und zu einer stärkeren Ausbreitung beitragen können. Hierbei können Strategien im Umgang mit Infektionskrankheiten, die Verdrängung und Unterdrückung von Ängsten beinhalten, einen Teufelskreis auslösen, wobei Ängste und Verdrängung sich gegenseitig verstärken.

Schlussfolgerungen Die COVID-19-Pandemie stellt eine immense Herausforderung mit noch ungewissem Ausgang für Regierungen, Gesundheitssysteme und Menschen dar, die mit erheblichen Beeinträchtigungen der psychischen Gesundheit in der Bevölkerung verbunden ist. Im Einklang mit Empfehlungen der WHO sollten nationale Leitlinien und präventive Maßnahmen die psychischen Auswirkungen, die Akzeptanz und Normalisierung von Ängsten und die Förderung von Resilienz in der Bevölkerung im Umgang mit COVID-19 umfassen, um einer weiteren Ausbreitung entgegenwirken zu können.

\section{ABSTRACT}

Aim The aim of the article is to point out the important role of prevention and reduction of mental stress in the general population and in sensitive groups in the context of the coronavirus disease 2019 (COVID-19) pandemic.

Methods This article includes the analysis and evaluation of studies and recommendations from organizations such as the World Health Organization (WHO) that have examined the psychological consequences of epidemics/pandemics on people and their impact on the further course.

Results Fear-related behaviors can adversely affect the course of epidemics. Past outbreaks of infectious diseases (Ebola and Zika virus) have shown that maladaptive behavior, related to increased psychological stress and anxiety, can interfere with the implementation of treatment strategies and actions and can contribute to a further spread. Hereby, strategies for dealing with infectious diseases, that include the suppression 
of fear, can trigger a vicious circle in which fear and suppression mutually reinforce each other.

Conclusion The COVID-19 pandemic poses an immense challenge to governments, health systems and people, with an uncertain outcome, which is associated with a significant burden of mental health in the population. In line with WHO rec- ommendations, national guidelines and preventive measures should include the psychological consequences, the acceptance and normalization of fears and the promotion of resilience in the population in dealing with COVID-19 in order to counteract a further spread.
Bundeskanzlerin Angela Merkel bezeichnete die Coronavirus-Krankheit-2019 (COVID-19) als die größte Herausforderung seit dem Zweiten Weltkrieg. Am 11. März 2020 erklärte die Weltgesundheitsorganisation (WHO) den COVID-19-Ausbruch zur Pandemie, die mittlerweile mindestens 208 Länder/Regionen betrifft, wobei die Zahl der bestätigten Infektionen bei mehr als 1 Million bzw. der Todesfälle bei über 60000 liegt (Stand: 5. April 2020) [1]. Während der Ausbruch in Wuhan in der chinesischen Provinz Hubei begann, ist mittlerweile der Großteil der bestätigten Infektionen und Todesfälle außerhalb des Landes zu verzeichnen, wobei sich hier Italien, Spanien und die USA als Epizentren herauskristallisieren. COVID-19 wird durch ein neuartiges Coronavirus (SARS-CoV-2), ein umhülltes einzelsträngiges RNA-Virus, verursacht, wobei nun insgesamt 7 humanpathogene Coronaviren bekannt sind. Im Gegensatz zu anderen Coronaviren, die hauptsächlich eine gewöhnliche Erkältung auslösen wie z. B. 229E, OC43, NL63 und HKU1, ähnelt das SARS-CoV-2 dem SARS-assoziierten Coronavirus (SARSCoV) aus dem Jahr 2002 und dem MERS-assoziierten Coronavirus (MERS-CoV) von 2012, die in einer akuten infektiösen Lungenentzündung münden können. Ähnlich wie bei SARS und MERS weisen bisherige Untersuchungen auf Fledermäuse als Ursprung von SARSCoV-2 hin (89-96\% Nukleotididentität), das von einem Zwischenwirt (vermutlich ein Pangolin - 91 \% Nukleotididentität) auf den Menschen überging [2].

Die weltweit immer weiterwachsenden Infektionszahlen und die immensen Herausforderungen mit denen Regierungen, Gesundheitssysteme und Menschen konfrontiert sind, führen in der Bevölkerung zu erheblichen Beeinträchtigungen der psychischen Gesundheit. Kognitive und emotionale Stressreaktionen, die mit dem Ausbruch einer Infektionskrankheit einhergehen, können dabei das ständige Grübeln über die eigene Gesundheit und die Gesundheit von Familienangehörigen und Freunden, Angst und Unruhe sowie das Gefühl von Einsamkeit oder Isolation umfassen. Dies kann sich auch in körperlichen und Verhaltenssymptomen wie diffuser Schmerz, Schlafstörungen, Alkohol- und Drogenkonsum sowie selbstverletzendem oder suizidalem Verhalten niederschlagen. Insbesondere können Maßnahmen zur Verlangsamung der Ausbreitung von COVID-19 wie soziale Distanzierung, Ausgangssperren, Reiseverbote, Schließung von Unternehmen und öffentlichen Einrichtungen sich stark auf das alltägliche Zusammenleben, übliche Aktivitäten und Routinen der Menschen auswirken. Besonders betroffen sind sensible Gruppen wie Gesundheitsdienstleister, ältere Menschen mit chronischen Krankheiten, Kinder und Jugendliche sowie Menschen mit psychischen Erkrankungen, bei denen ein höheres Risiko besteht für die Entwicklung von Angststörungen, depressiven Störungen und posttraumatischen Belastungsstörungen. Besonders wichtig ist hier, dass bei Menschen mit chronischen und psychischen Krankheiten sowie Frontarbeitern konkrete Maßnahmen ergriffen werden müssen, die körperliche und psychische Belastungen verringern. In diesem Sinne ist es unerlässlich, dass Menschen mit bestehenden Erkrankungen ihre Behandlungen fortsetzen können, um das Auftreten neuer oder sich verschlechternder Symptome zu verhindern oder dass Frontarbeiter, bei denen ein höheres Infektionsrisiko besteht, hinreichende Unterstützung erhalten. Ergebnisse früherer Untersuchungen haben gezeigt, dass Epidemien und v. a. Pandemien den wahrgenommenen Stress in der Gesamtbevölkerung signifikant erhöhen und dass die Verfügbarkeit politischer und gesellschaftlicher Bewältigungsmöglichkeiten invers mit der Häufigkeit von Epi-/Pandemieereignissen assoziiert ist [3]. Darüber hinaus hat der Ausbruch des Ebola-Virus aus dem Jahr 2014 gezeigt, dass angstbedingte Verhaltensweisen eine wichtige Rolle für den weiteren Verlauf einer Epidemie spielen können [4]. Hierbei wurde gezeigt, dass maladaptive Verhaltensweisen bedingt durch erhöhte psychische Belastungen und Ängste die Implementierung von Behandlungsstrategien und -maßnahmen beeinträchtigen und zu einer stärkeren Ausbreitung beitragen. Die Autoren schlussfolgerten, dass zukünftige Maßnahmen, die darauf abzielen die Ausbreitung von Epidemien zu verhindern, das Auftreten und die Aufrechterhaltung von angstbedingten und maladaptiven Verhaltensweisen mitberücksichtigen sollten, um Resilienz zu fördern und Schaden abzuwenden. Demzufolge haben China und andere Länder psychologische Kriseninterventionen eingeführt, um die psychischen Belastungen in der Bevölkerung zu reduzieren und die Widerstandsfähigkeit gegenüber solchen Krisen zu erhöhen [5]. Ein wichtiger Bestandteil solcher Maßnahmen sollte, unabhängig von der exakten Gestaltung, die Akzeptanz und Normalisierung von Ängsten und negativen Emotionen darstellen, da akuter psychischer Stress bei Konfrontation mit einem Ereignis solchen Ausmaßes eine normale Reaktion ist. So konnte im Rahmen des Ausbruchs des ZikaVirus ermittelt werden, dass die Verdrängung und Unterdrückung von Ängsten einen Teufelskreis auslösen kann, wobei der Einsatz von Verdrängung die Intensität der Angstreaktionen erhöhen kann [6]. Da die überintensive Beschäftigung mit beunruhigenden Medieninhalten sowie mit Fehlinformationen in sozialen Medien gepaart mit der teilweise mangelnden Kompetenz der Allgemeinbevölkerung gesundheitsbezogene Information adäquat zu bewerten bedeutsame Angstquellen darstellen, ist es überaus wichtig, zuverlässige, akkurate und verständliche Informationen bereitzustellen, um der Angst entgegenzuwirken. Im Allgemeinen sollten stressreduzierende Interventionen auf die Aufrechterhaltung von sozialen Kontakten, Verringerung von Stigmatisierung und Diskriminierung im Zusammenhang mit der Epidemie, Aufrechterhaltung von Normalität und eines gesunden Lebensstils sowie Nut- 
zung von psychosozialen Unterstützungssystemen über telefonische und internetbasierte Beratungssysteme fokussieren [5].

Psychische Belastungen in der Bevölkerung als Reaktion auf einen unvorhersehbaren Verlauf im Rahmen einer Epidemie führen möglicherweise nicht nur zu einer Zunahme von angstbedingten Verhaltensweisen, Symptomen und Störungen, sondern schränken auch unmittelbar die gesamtgesellschaftlichen Fähigkeiten für einen adäquaten Umgang mit Epidemien ein, wodurch Infektionsraten und Todesfälle weiter steigen. Daher sind Maßnahmen notwendig, wie auch von der WHO und anderen Gesundheitsorganisationen empfohlen, die sich spezifisch mit den negativen Auswirkungen im Hinblick auf die psychische Gesundheit in der Bevölkerung befassen, insbesondere bei sensiblen Gruppen wie Frontarbeitern. Diese Aspekte sollten in die nationalen Leitlinien und Empfehlungen integriert werden mit dem Ziel die Resilienz der Bevölkerung zu stärken und um die Ausbreitung der COVID-19-Pandemie zu stoppen [7].

\section{Interessenkonflikt}

Die Autorinnen/Autoren geben an, dass kein Interessenkonflikt besteht.

\section{Literatur}

[1] World Health Organization, 2020. https://experience.arcgis.com/exper ience/685d0ace521648f8a5beeeee1b9125cd (zuletzt aufgerufen am 5. April 2020)

[2] Clerkin KJ, Fried JA, Raikhelkar J et al. Coronavirus Disease 2019 (COVID-19) and Cardiovascular Disease. Circulation 2020. doi:10.1161/CIRCULATIONAHA.120.046941. doi:10.1161/CIRCULATIONAHA. 120.046941

[3] Pan American Health Organization. Protecting Mental Health During Epidemics, 2006. https://www.paho.org/en/documents/protectingmental-health-during-epidemics (zuletzt aufgerufen am 27. März 2020)

[4] Shultz JM, Cooper JL, Baingana F et al. The Role of Fear-Related Behaviors in the 2013-2016 West Africa Ebola Virus Disease Outbreak. Curr Psychiatry Rep 2016; 18: 104. doi:10.1007/s11920-016-0741-y

[5] Bao Y, Sun Y, Meng S et al. 2019-nCoV epidemic: address mental health care to empower society. Lancet 2020; 395: e37-e38. doi:10.1016/S0140-6736(20)30309-3

[6] Dillard JP, Yang C, Li R. Self-regulation of emotional responses to Zika: Spiral of fear. PLoS One 2018; 13: e0199828. doi:10.1371/journal. pone. 0199828

[7] World Health Organization. Mental health and COVID-19, 2020. http:// www.euro.who.int/en/health-topics/health-emergencies/coronaviruscovid-19/novel-coronavirus-2019-ncov-technical-guidance/ coronavirus-disease-covid-19-outbreak-technical-guidance-europe/ mental-health-and-covid-19 (zuletzt aufgerufen am 28. März 2020) 